\title{
Explanation in Classical Population Genetics
}

\author{
Anya Plutynskitł
}

The recent literature in philosophy of biology has drawn attention to the different sorts of explanations proffered in the biological sciences - we have molecular, biomedical, and evolutionary explanations. Do these explanations all have a common structure or relation that they seek to capture? This paper will answer in the negative. I defend a pluralistic and pragmatic approach to explanation. Using examples from classical population genetics, I argue that formal demonstrations, and even strictly "mathematical truths," may serve as explanatory in different historical contexts.

1. Introduction. The topic of this symposium is the making of a theoryin particular, the making of the "genetical" theory of evolution via the early synthesis of Mendelian genetics with Darwinism in the work of Fisher, Haldane, and Wright in the 1920s and 1930s. Scientific theories are supposed to be about the world. Yet, classical population genetics is a formal discipline treating not so much the world as possible worlds. The simple one-locus, two-allele models of Fisher, Haldane and Wright sweep aside the "disorderly complexity" of genetic interaction, development and changing environments for the sake of the "meaningful simplicity" of idealized relationships between population size and effects of selection, mutation, and drift on distributions of gene frequency. ${ }^{1}$ Richard Lewontin (2000) has called the achievements of classical population genetics "minimal deductive programs," purely analytic statements about the interactive effects of population size, mutation, migration, etc. in pop-

$\dagger$ To contact the author, please write to: Department of Philosophy, University of Utah, 260 S. Central Campus Drive, Orson Spencer Hall, Room 341, Salt Lake City, UT 84112; e-mail: plutynski@philosophy.utah.edu.

\$Thanks to my fellow symposiasts: Margaret Morrison, Sahotra Sarkar, and Rob Skipper for joining, and to John Beatty for chairing. Thanks also to Kyle Stanford for his questions and comments.

1. According to Herbert Simon, "The purpose of science is to find meaningful simplicity in the midst of disorderly complexity" (cited in Crow 2001).

Philosophy of Science, 71 (December 2004) pp. 1201-1214. 0031-8248/2004/7105-0048\$10.00 Copyright 2004 by the Philosophy of Science Association. All rights reserved. 
ulations represented strictly in terms of allele frequencies. From the perspective of today's advances in molecular and developmental biology, this may seem a hollow achievement. One may well ask what value there is in predicting the future state of an infinite population consisting of noninteracting genes with single phenotypic effects, each assigned constant selection coefficients, as there could be no such populations!

In part due to these sorts of 'possible worlds' explanations, classical population genetics has been one of the more maligned subdisciplines of biology. Punnett complained of Fisher's 1918 groundbreaking paper, "On the Correlation between Relatives on the Supposition of Mendelian Inheritance," that it treated of "weightless elephants on frictionless surfaces" (Punnett, [1916] 1976). Ernst Mayr famously christened population genetics "beanbag genetics" - insofar as it treats evolution as mere changes in gene frequency, as if evolution were nothing more than the "adding and removal of beans from a bag" (Mayr, 1959). And Will Provine writes in the new afterward to his history of the Origins of Theoretical Population Genetics:

In 1970 I could see the origins of theoretical population genetics as being an unalloyed good for evolutionary biology, and thus obviously a great subject for an historian. Now I see these same models of the 1930 's, still widely used today, as an impediment to the understanding of evolutionary biology, and their amazing persistence in textbooks and classrooms as a great topic for other historians. ([1971] 2001, 204)

How to explain this shift? According to Provine, classical population genetics today does not so much improve as it impedes understanding of evolution. Classical population genetics, in treating evolution as mere change in gene frequency, is "reductionistic," committed to genic selectionism, or unabashedly dismissive of the significant factors of genetic interaction and development, or so say some of its most ardent critics.

And yet, despite its beanbag idealism and reductionism, population genetics, in the first twenty-five years of the twentieth century, changed the face of biology, or so I will argue. ${ }^{2}$ Work by Haldane, Fisher, and Wright gave biologists a reason to adopt a Darwinian worldview, and to

2. Some (Smocovitis, 1996) might question my historical claim here as follows. Perhaps this was not a scientifically motivated shift so much as an ideological shift. Biologists employed the rhetoric of synthesis, some say, for reasons having to do not with science but politics. While I acknowledge that there were institutional and political forces at work in the synthesis (see Cain 1994), I wish here to focus on the scientific contributions of some of the early architects, where "scientific" is construed broadly so as to countenance formal and empirical science. 
view Darwinism and Mendelism as compatible and complimentary. How was this possible? How could mathematical models that treat of (strictly speaking) fictional states of affairs so alter the history of biology? How can false models explain? My answer, in part, comes from examination of the historical context, and in part, from a philosophical reconsideration of the role of the formal sciences and of mathematical models in scientific explanation.

First, a bit of historical context and a caveat. Haldane, Fisher, and Wright saw themselves as providing a refutation of neo-Lamarckian, orthogenetic, and generally anti-Darwinian views. It is often forgotten that at the time Haldane, Fisher, and Wright were beginning their scientific careers, Darwinism (conceived as the commitment to natural selection being a major cause in generating the diversity of life) was widely viewed to be discredited by biologists both in Europe and the U.S. (see Bowler 1983). One of the first books Wright read as a student of biology, Kellogg's Darwinism To-day, holds that, "Darwinism, as the all-sufficient, or even the most important causo-mechanical factor in species-forming and hence as the sufficient explanation of descent, is discredited and cast down" (1903, 374). When Fisher, Haldane, and Wright first began their project of developing a mathematical biology, each of them was well aware of this general concern, and so, had a common set of enemies. Using simple mathematical models, they wished to demonstrate the compatibility of a Mendelian system of inheritance and Darwinism, and the tenability of a Darwinian research program. To the extent that they were successful, it is incumbent upon historians and philosophers of science to address the following questions:

1. How did Haldane, Fisher, and Wright achieve their aims?

2. More generally, how are idealized formal models such as those of classical population genetics explanatory?

The object of this paper will be to respond to these questions. More precisely, I will defend the following three theses. First, the standard approaches to the problem of scientific explanation are insufficient to account for the ways in which the formal models of classical population genetics explain. Second, the best way of making sense of the role that these models play in answering requests for explanation is appeal to van Fraassen's pragmatic approach. Third, some examples from classical population genetics may serve as a case in point for how the same model may explain at several levels, or relative to several different contrast classes that are salient in different historical contexts (van Fraassen 1980).

Let me first issue a caveat. I do not mean to suggest here that Haldane, Fisher, and Wright single-handedly refuted the anti-Darwinians. Indeed, as Beatty (1986) and others (Provine [1971] 2001) have pointed out, this 
shift occurred gradually and was the result of efforts of both an empirical and a formal sort. I simply wish to claim here that the formal demonstrations were explanatorily relevant to the refutation of anti-Darwinism.

2. Standard Approaches to Explanation. For Hempel (1965), the aim of a philosophical theory of scientific explanation is normative; we are to identify the structure and conditions of adequacy of a scientific explanation. Once we have identified this common structure or set of conditions, we may demarcate good from bad, scientific from nonscientific explanations. Hempel's solution to the problem was a family of deductive models; according to Hempel, explanations are arguments with sets of laws and initial conditions constituting the explanans, and facts or events to be explained in the explananandum. On this account, explanations run parallel with predictions.

However, the explanations made possible by appeal to the models of population genetics do not run parallel with predictions; i.e., they are not if-then arguments with specific facts or events in the consequent. Rather, the explananda in population genetics are not facts or events in the world, in the sense of propositions about what is the case. Rather, what is explained is what is possible, and what is necessary, given certain assumptions about the genetics of populations. Hempel may have called such explanations explanation-sketches.

The other two "venerable traditions" of thinking about scientific explanation take it that the aim of a philosophical theory of explanation is not so much normative as descriptive. We are to look closely to the practice of science and see what it is that all explanations in science share. ${ }^{3}$ On the one hand, Salmon has defended the view that scientific explanations provide information about the causal structure of the world (1984). On the other hand, Kitcher defends the view that a scientific theory is explanatory when it unifies several phenomena under a common framework, or family of argument schemata (1989).

However, in my view, neither of these two traditions quite captures the way in which mathematical models in biology provide explanatory information. First, with respect to the causal model, as Sober (1983) has pointed out, equilibrium models such as Fisher's sex-ratio model are not causal per se, insofar as they do not identify specific causal factors at work in any particular case of a population at equilibrium. Instead, equilibrium models present us with disjunctions of possible causal scenarios. Sober contends that giving the actual causal history of some population may be less explanatory than a general demonstration of the sort Fisher 
provided. Indeed, he claims that "once an evolutionary model is stated carefully, it often turns out to be a nonempirical mathematical truth" $(1999,72)$. If mathematical truths are explanatory, then we certainly need to rethink the causal model of explanation!

Second, the unificationist approach of Kitcher, while bringing out attention to one important virtue of scientific explanation, does not cover all cases. Sometimes scientists may wish for a causal explanation of some particular facts or event. Sometimes they may wish to identify a class or kind of event, or see a common pattern across disparate events. And sometimes, they may wish for an explanation of what's possible, given some set of constraints. In these cases, what will count as an explanation may consist of a mathematical demonstration or a proof of what must be so, for any population that fits (ceteris paribus) the description the model. In my view, the explanatory value of these sorts of mathematical demonstrations has been all but ignored by philosophers of science (but see Glymour 1980).

While I think that each of the several ways in which philosophers of science have attempted to capture the explanatory relation is promising in one or another context, it's not clear that their identification of the explanatory relation as one of causation, unification, or even the latest candidates of invariance or stability, is sufficient to account for the several ways in which scientists explain. Indeed, I think it's a mistake to look for one virtue in which scientific explanations are explanatory - either in identifying the cause, relation of stability or invariance, or what have you. Philosophers of science have, I think, been mistaken in seeking out the relation in virtue of which scientific explanations explain. So, while the pluralist approach would certainly be unsatisfying if what we are looking for is a solution to Hempel's problem, it seems that it is unavoidable if what we are in search of it a descriptively adequate account of scientific explanation.

3. An Alternative Approach. In this section I will defend a pluralistic and pragmatic approach to scientific explanation, one which I believe to be more adequate to scientific practice. One way of understanding explanatory pluralism is the following. Scientific understanding admits of kinds and degrees. Once we grant that there are many types of scientific understanding, perhaps we ought also to grant that there are many types and virtues of scientific explanations. I take Salmon (1990) to have defended a view akin to this when he claimed that scientific explanations may satisfy two sorts of desires for understanding: gaining a unified worldview, or Weltanschauung, and opening the black box and finding out how 
things work. ${ }^{4}$ Salmon seems to have gone some way towards supporting the view here that the enterprise of explaining in the sciences is not a single kind of activity.

The explanatory role of models and mathematical demonstration in biology is not restricted to demonstrating how a change in one variable yields a change in another. There is another class of explanations that draw upon mathematical modeling that is worth examining. Sometimes, a scientific community may wish to know why we ought to adopt this research program rather than another, or whether two research programs are compatible. In service of answering these latter sorts of questions, scientists may make appeal to formal mathematical demonstrations, or proofs of possibility. Whether or how such a demonstration is explanatory will depend importantly on the historical context.

Van Fraassen's (1980) account of the pragmatics of explanation serves as a systematic framework for making this last point. According to van Fraassen, theories of explanation have mistakenly been understood to be two-term relations between theory and fact, when indeed they are threeterm relations between theory, fact, and context:

Being an explanation is essentially relative, for an explanation is an answer. Since an explanation is an answer, it is evaluated vis-a-vis a question, which is a request for information. But exactly what is requested differs from context to context. . . . the background theory plus data relative to which the question is evaluated as arising or not arising, depends on the context. . . . And, even what part of that background information is to be used to evaluate how good the answer is, qua answer to that question, is a contextually determined factor. (van Fraassen 1980, 153)

I take van Fraassen's point in the above passage to be the following. Historical context and the interests of the questioner will determine what counts as an interesting and important question, and relatedly, what

4. Another respect in which one may be a pluralist about scientific explanation is to suggest that one event can be correctly explained in two ways (Grantham 1999). I will not be defending this view here. 
counts as good answer. ${ }^{5}$ I think that this general point may help us make sense of Provine's claim above that the development of classical population genetics, while once apparently an unalloyed good for evolutionary biology, today seems an impediment to understanding. At an early stage in some science, our background theory plus data may lead us to conclude that we have two different research programs that are intrinsically at odds with one another. So, whether it is possible that known data accounted for on one hypothesis could be accounted for on another may be a question of great import. Such questions as "How is it possible that this could come about?" or "How can we conceive of these two theories or hypotheses as compatible?" are not insignificant questions at certain stages of scientific inquiry. Constructing models is one very effective way of answering these sorts of questions. Moreover, the same demonstration may answer two requests for explanation simultaneously; such demonstrations may both explain how it is possible that known phenomena could be accounted for on this or that theory, and why we may have good reason to adopt a new research program that takes such a theory as central. Of course, whether we regard such demonstrations as explanatory in these ways is importantly contingent upon historical context.

4. Cases in Point. Let us turn to two examples from the history of classical population genetics that serve as cases in point. The first is drawn from Fisher's 1918 paper, "The Correlation between Relatives on the Supposition of a Mendelian Inheritance," and the second is drawn from Haldane's 1924 paper, "A Mathematical Theory of Artificial and Natural Selection." Each of these simultaneously answered specific how-possibly questions, but also offered proofs of possibility, on the one hand, of the compatibility of Darwinism with a Mendelian theory of inheritance, and on the other, of the possibility of relatively weak selection generating significant change in a short amount of evolutionary time. Moreover, the

5. While one may be moved by Kitcher and Salmon's (1987) objection here that this view may seem to countenance an "anything goes" stance on explanation, according to which there may be no objective standards for what counts as explanatory, Lloyd and Anderson (1993) and Richardson (1992) have pointed out that van Fraassen has a reply to such objections, even if to some it may sound like a slippery one. Roughly, his answer is that what we may rule out as nonscientific will depend upon our best science. While this may seem unsatisfactory for one who thinks that for an explanation to be scientific it must be true, if my examples are any guide, it seems that possibility and not only truth is of interest to scientists. I agree with van Fraassen that an answer to what sorts of explanations count as explanatory is not a question that philosophers may expect to answer a priori, but that the answer to this question will depend importantly on the scientific community's standards, their background theories, etc. (But see also Woody 2004.) 
demonstrations both rested on, strictly speaking, false assumptions, and the conclusion, at least in Fisher's case, depended entirely upon his presuppositions. Formal mathematical demonstrations of this sort, I wish to argue, are explanatory in that they are effective at providing proofs of possibility. Sober has said that our evolutionary models often provide, strictly speaking, arguments where, once all the premises are set out, the conclusion follows necessarily; they are, he says, "mathematical truths." How can these mathematical truths be explanatory? Here is how.

Fisher's 1918 paper is an attempt to demonstrate how a multifactorial theory of inheritance can serve to underpin observed correlations between relatives discovered through biometrical methods. Thus it is widely regarded as the seminal paper which successfully synthesized two hitherto distinct and opposing schools on the science of heredity and evolution, biometry and Mendelism. For our purposes, we can take the results of biometry to be the following; the vast majority of continuous traits (height, weight) exhibit a normal distribution, or if not, can be broken down into sums of two or more normal distributions. Correlations between relatives may be established, and these correlations decrease geometrically relative to ancestors. The biometricians had done exhaustive measurements of a range of continuous traits and found quantitative correlations between relatives for human height, weight, length of forearm, etc. Moreover, they were Darwinians in the classical sense; they believed that evolution was gradual, or that small selective effects of minute variations already present in natural populations were sufficient to explain the diversity of life we see today. In contrast, the Mendelians, who tended to study distinct variations that behaved in a Mendelian fashion (complete dominance, independent assortment, Mendelian ratios, etc.), held that evolution must take place via major transitions. For example, de Vries believed that mutations of large effect generated entirely new species. Fisher's ostensive project was to demonstrate that, given a number of assumptions, the observed correlations between various pairs of relatives, father-son, cousin, and siblings, among others, can be explained on the presupposition that traits such as height are derivative from a multitude of Mendelian, or discrete, independent factors. His more general goal was to demonstrate the compatibility of biometry and the Darwinian perspective with Mendelism; i.e., that the gradualist picture of evolution was not antithetical to the new particulate theory of inheritance, as many believed.

As with almost all of Fisher's theoretical work, his assumptions were idealized and crucial to his conclusions. ${ }^{6}$ What made his demonstration

6. However, his analysis of the correlation was not wholly idealized; he successively dealt with a number of realistic biological complications: complete or partial recessivity, multiple alleles, epistacy, linkage, and assortative mating. He showed that with each 
possible was that he relaxed some of the assumptions that others were unwilling to give up. This is exactly what Fisher meant when he wrote in the first lines of the paper that "it is here attempted to ascertain the biometrical properties of a population of more general type than had hitherto been examined." In particular, he assumed that dominance was not complete, but that the heterozygote could be at any point intermediate between homozygotes. Fisher also assumed that the relevant Mendelian factors were entirely independent in their effect, and that the number and effects of such factors affecting any particular trait was quite large. Starting with these assumptions, he showed how a normal distribution of measurements of some trait may follow from a particulate scheme of inheritance.

Let us follow Fisher's reasoning. First, he comments on the fact that the correlations found between relatives when Pearson and Yule earlier attempted to follow through with the consequences of a particulate theory were too small, in part resulting from their assumptions of complete dominance. Fisher suggests that we relax this assumption, and suggests alternatively that we assume that dominance is not absolute, but that the heterozygote may occupy an intermediate state between homozygotes. Next, he asks us to suppose that the measurement of some trait is the sum of the effects of a large number of independent factors. If the factors underpinning a trait were sufficiently numerous, acted independently, and were minute in their effects, then they would yield a normal distribution. This follows necessarily from the central limit theorem. As Moran and Smith have written:

The fact that the distribution of the sum of all factors will be approximately normally distributed will follow from a version of the Central Limit theorem, which proves asymptotic normality for a sum of independent random variables each of which is "individually negligible" in their effects. $(1966,6)$

By ignoring complications such as the extent and nature of dominance, whether the "factors were coupled" (whether there was linkage of genes on the chromosome), as well as preferential mating, selection, and effects of the environment, Fisher arrives at the normal distribution. He later commented that his method here was analogous to that of Maxwell in deriving the velocity distribution of molecules in a gas:

added complication, the correlation between relatives remained compatible with the empirical results of the biometricians. This paper illustrates Fisher's persistent method of beginning with a great deal of abstraction, and then gradually showing how, even with the added complication of biological reality, the effects still "average out." 
the whole investigation may be compared to the analytical treatment of the Theory of Gases, in which it is possible to make the most varied assumptions as to the accidental circumstances, and even the essential nature of the individual molecules, and yet to develop the general laws as to the behavior of gases, leaving but a few fundamental constants to be determined by experiment. (1922, 331-332).

Maxwell assumed for the theory of gases that the molecules of a gas are numerous, minute, perfectly elastic, and have no interactive effects. Analogously, Fisher assumes for his genetical theory that the hereditary factors were sufficiently numerous, acted independently and were minute in their effects.

However, the power of Fisher's demonstration of the compatibility of biometry with the particulate theory did not end with the demonstration that a normal distribution followed from the assumptions mentioned. He used the analysis of variance to partition the causes of variation in a trait into dominance and additive components. Next, he demonstrated how the additive component of the variance contributed to the correlation between relatives. Thus, he showed how, on a particulate theory, the correlations between relatives were approximately those given by the biometricians, and these correlations could be due to the additive component of the variance. ${ }^{7}$ What Fisher did was not so much add to the number of facts explained as reconceive the object of explanation. A population of organisms was, in a sense, reduced to a cloud of points with no interactive forces and independent actions. The normal distribution and the correlations between relatives followed from these assumptions. Fisher thus showed how a very attenuated sense of Mendelism was compatible with the results of biometry.

Fisher's demonstration answered two "how possibly" questions simultaneously: How is it possible that the known correlations between relatives could be determined by discrete genetic factors? And, how is Darwinism compatible with Mendelism? This example can help us make sense of Sober's claim mentioned above that many of the mathematical models in evolutionary biology are, once examined closely, nonempirical mathematical truths, but are nonetheless explanatory. Often, following through the mathematical consequences of one's assumptions can be explanatory insofar as one shows how something is possible, given some set of suppositions.

Similarly, the mathematical demonstrations of Haldane in his 1924 paper of the effectiveness of small selection coefficients over a relatively short amount of time serve as a second case in point of how a model 
provides explanatory information of a "how possibly" sort. His mathematical demonstration of how, given an initial set of conditions, selection affects the distribution of alleles in a population both explained the effects of selection in a diploid population and showed why it is not implausible that selection can generate significant change over a short span of "geological" time. Note that his explanandum here was not some fact or event, such as the evolution of the vertebrate limb. Rather, he was simply describing the mathematical consequences of a small selection coefficient on the distribution of genes in a population over time - essentially deriving a logistic curve.

The cases above serve as evidence for my pluralist and pragmatist thesis. A mathematical demonstration may serve to address both more specific as well as general concerns simultaneously. The same mathematical model can provide explanatory information about how two theoretical frameworks are related and about whether we ought to adopt this or that research program. We are mistaken when we look to classical population genetics exclusively to explain particular events or states of affairs in the world. It's better to view population genetics as either providing explanations of classes of events or processes, or providing proofs of possibility. Thus, there are several virtues in which a theory or model may serve as explanatory; it may provide us with a novel Weltanschauung, or "open up the black box," or it may provide us with demonstrations of possibilities. Such proofs may be of great relevance at certain stages in science. Today, with the vast amount of empirical evidence of the effectiveness of selection (see, e.g., Endler 1986) we may well be unimpressed by Haldane's demonstration. However, in 1924, it was quite significant. I think that an appeal to the context sensitivity of requests for explanation can help us make sense of Provine's comment cited above. He was correct in 1970 that the development of classical population genetics was an unalloyed good for evolutionary biology, and he may well be right that today it impedes understanding.

\section{Appendix: The Correlation between Relatives}

Fisher's own demonstration that the correlations between relatives found by biometricians was explainable on the supposition of a Mendelian or particulate scheme of inheritance is too technically difficult to explain here. I derive the following mathematical demonstration from Ewens's (1979).

Consider a trait that is determined entirely by a locus with two alleles, $\mathrm{A} 1$ and A2. Suppose that all A1A1 individuals have measurement $m_{11}$ for 
TABLE 1.

\begin{tabular}{lcccc}
\hline \multirow{2}{*}{$\begin{array}{c}\text { GENOTYPE: } \\
\text { FATHER }\end{array}$} & Phenotype & A1A1 & A1A2 & A2A2 \\
\cline { 2 - 5 } A1A1 & $m_{11}$ & $x^{3}$ & $x^{2}(1-x)$ & 0 \\
A1A2 & $m_{12}$ & $x^{2}(1-x)$ & $x(1-x)$ & $x(1-x)^{2}$ \\
A2A2 & $m_{22}$ & 0 & $x(1-x)^{2}$ & $(1-x)^{3}$ \\
\hline
\end{tabular}

the character, and all $\mathrm{A} 1 \mathrm{~A} 2$ have measurement $m_{12}$, etc. Let us assume no environmental contributions to expression of the phenotype.

Suppose random mating obtains, such that the frequencies of A1A1, $\mathrm{A} 1 \mathrm{~A} 2$, and A2A2 are $x^{2}, 2 x(1-x)$, and $(1-x)^{2}$. The mean value of the measurement of this trait will then be:

$$
M=x^{2} m_{11}+2 x(1-x) m_{12}+(1-x)^{2} m_{22} .
$$

And the variance will be:

$$
\sigma^{2}=x^{2}\left(m_{11}-M\right)+2 x(1-x)\left(m_{12}-M\right)^{2}+(1-x)^{2}\left(m_{22}-M\right)^{2} .
$$

What is the covariance between father and son for the measurement of this trait? Suppose the father is A1A1. The son will be either A1A1 with probability $x$ or A1A2 with probability $(1-x)$. The father is A1A1 with probability $x^{2}$. It is possible to draw up a table of the probabilities of various father-son combinations (see table 1). The covariance between the father and son is thus:

$$
\begin{gathered}
x^{3} m_{11}^{2}+2 x^{2}(1-x) m_{11} m_{12}+x(1-x) m_{12}^{2}+2 x(1-x)^{2} m_{12} m_{22} \\
+(1-x) 3 m_{22}^{2}-M^{2}=x(1-x)\left\{x m_{11}+(1-2 x) m_{12}-(1-x) m_{22}\right\}^{2} .
\end{gathered}
$$

The correlation between the two measurements is found by dividing the covariance by the variance:

$$
x(1-x)\left\{x m_{11}+(1-2 x) m_{12}-(1-x) m_{22}\right\}^{2} / \sigma^{2} .
$$

Here it will be useful to introduce two new concepts, additive and dominance variance. Let the total variance $\sigma^{2}$ be made up of two components, the additive and nonadditive:

$$
\begin{aligned}
& \sigma_{A}^{2}=2 x(1-x)\left\{x m_{11}+(1-2 x) m_{12}-(1-x) m_{22}\right\}^{2}, \\
& \sigma_{D}^{2}=x^{2}(1-x)^{2}\left\{2 m_{12}-m_{11}-m_{22}\right\}^{2} .
\end{aligned}
$$

One can see that the total variance in the measurement of a trait is a sum of the additive and dominance variance. The genic or additive variance is that part of the total variance in a character which can be accounted for by the average effect of the substitution of the A1 allele for the A2 allele at the locus. (Fisher used a least squares method in order to dem- 
onstrate average effect of substitution of an allele. I will not describe the method here, as it involves some tricky mathematics.) Suffice it to say that we can partition the variance in the measurement of a trait into two parts, the additive and the dominance, or residual variance. The residual variance describes the excess in the case that the heterozygote is not intermediate between each homozygote. Or, it is just that part of the variance in excess of the additive, when:

$$
m_{12} \text { does not exactly equal } \frac{1}{2}\left(m_{11}+m_{22}\right) .
$$

The expression above for the covariance may thus be rewritten as follows:

$$
\frac{1}{2} \sigma_{A}^{2} / \sigma^{2}
$$

Thus, the above demonstrates that the correlation between father and son will be half the ratio of the additive genetic variance to the total variance, or approximately $\frac{1}{2}$. Considering the case of full sibs, we get a correlation of approximately $\frac{1}{2}+\frac{1}{4}$ the ratio of dominance variance to total variance (thus accounting for the higher correlation between sibs), $\frac{1}{4} \sigma_{A}^{2} / \sigma^{2}$ for uncle and nephew, and $\frac{1}{4} \sigma_{A}^{2} / \sigma^{2}+\frac{1}{6} \sigma_{D}^{2} / \sigma^{2}$ dominance to total variance for double first cousins. Subsequent to the above demonstration, Fisher showed that with the added complication of many loci, as well as assortative mating, approximately the same correlations hold. Recall that Fisher assumed that there were no effects of environment, and no correlation in environment between father and son.

\section{REFERENCES}

Beatty, John (1986), "The Synthesis and Synthetic Theory", in William Bechtel (ed.), Integrating Scientific Disciplines. Dordrecht: Martinus Nijhoff Publishers.

Bowler, Peter J. (1983), The Eclipse of Darwinism: Anti-Darwinian Evolution Theories in the Decades around 1900. Baltimore: Johns Hopkins University Press.

Cain, Joseph (1994), "Ernst Mayr as Community Architect: Launching the Society for the Study of Evolution and the Journal 'Evolution'”, Biology and Philosophy 9: 387-427.

Crow (2001), "The Beanbag Lives On", Nature 409: 771.

Endler, John A. (1986), Natural Selection in the Wild. Monographs in Population Biology. Princeton, NJ: Princeton University Press.

Ewens, Warren (1979), Mathematical Population Genetics. Berlin: Springer Verlag.

Fisher, Ronald Aylmer (1918), "The Correlation between Relatives on the Supposition of Mendelian Inheritance", Transactions of the Royal Society of Edinburgh 52: 399-433. (1922), "On the Dominance Ratio", Proceedings of the Royal Society of Edinburgh 42: 321-341.

Glymour, Clark (1980), "Explanation, Tests, Unity and Necessity”, Nous 14: 31-50.

Grantham, Todd (1999), "Explanatory Pluralism in Paleobiology", Philosophy of Science 66 (Proceedings): S223-S236.

Haldane, John Burdon Sanderson (1924), "A Mathematical Theory of Natural and Artificial Selection. Part I", Transactions of the Cambridge Philosophical Society 23: 19-41. 
Hempel, Carl (1965), Aspects of Scientific Explanation and Other Essays in the Philosophy of Science. New York: Free Press.

Kellogg, Vernon L. (1903), Darwinism To-day: A Discussion of Present-Day Scientific Selection Theories. London: Bell.

Kitcher, Philip (1989), "Explanatory Unification and the Causal Structure of the World", in Philip Kitcher and Wesley C. Salmon (eds.), Scientific Explanation, Minnesota Studies in the Philosophy of Science vol. 13. Minneapolis: University of Minnesota Press, 410 505 .

Kitcher, Philip, and Wesley Salmon (1987), "Van Fraassen on Explanation", Journal of Philosophy 84(6): 315-330.

Lewontin, Richard (2000), "What Do Population Geneticists Know and How Do They Know It?", in Richard Creath and Jane Mainschein (eds.), Biology and Epistemology. Cambridge: Cambridge University Press.

Lloyd, Elizabeth, and Carl Anderson (1993), "Empiricism, Objectivity and Explanation", in Peter A. French, Theodore E. Ueling, and Howard K. Wettstein (eds.), Philosophy of Science, Midwest Studies in Philosophy, vol. 18. Notre Dame, IN: University of Notre Dame Press, 121-131.

Mayr, Ernst (1959), "Where Are We?", Cold Spring Harbor Symposium on Quantitative Biology 24: 409-440.

Moran, Patrick Alfred Pierce, and C. A. B. Smith (1966), "Commentary on R. A. Fisher's Paper on the Correlation between Relatives on the Supposition of Mendelian Inheritance", Eugenics Laboratory Memoirs 41: 1-62.

Provine, William B. ([1971] 2001), The Origins of Theoretical Population Genetics. Chicago: University of Chicago Press.

Punnett, Reginald ([1916] 1976), report on Fisher's paper, Whittingham Lodge, Cambridge. Reproduced in Bernard Norton and E. S. Pearson, "A Note on the Background to, and Refereeing of, R. A. Fisher's 1918 Paper 'On the Correlation between Relatives on the Supposition of Mendelian Inheritance"', Notes and Records of the Royal Society of London, 31: 151-162.

Railton, Peter (1981), "Probability, Explanation, and Information", Synthese 48: 233-256.

Richardson, Robert (1992), "Explanation: Pragmatics and Asymmetry", Pacific Division, $A P A$.

Salmon, Wesley (1984), Scientific Explanation and the Causal Structure of the World. Princeton, NJ: Princeton University Press.

(1990), "Scientific Explanation: Causation and Unification", Critica revista hispanoamericana de filosofia 90: 3-24.

Smocovitis, Vassiliki Betty (1996), Unifying Biology: The Evolutionary Synthesis and Evolutionary Biology. Princeton, NJ: Princeton University Press.

Sober, Elliott (1983), "Equilibrium Explanation”, Philosophical Studies 43: 201-210. (1999), Philosophy of Biology, Dimensions of Philosophy Series. Boulder, CO: Westview Press.

van Fraassen, Bas (1980), The Scientific Image. Oxford: Clarendon Press.

Woody, Andrea (2004), "More Telltale Signs: What Attention to Representation Reveals about Scientific Explanation”, Philosophy of Science 71 (Proceedings): 780-793. 